\title{
Redundant Interdependencies Boost the Robustness of Multiplex Networks
}

\author{
Filippo Radicchi \\ Center for Complex Networks and Systems Research, School of Informatics and Computing, \\ Indiana University, Bloomington, Indiana 47408, USA
}

Ginestra Bianconi

School of Mathematical Sciences, Queen Mary University of London, London E1 4NS, United Kingdom (Received 17 October 2016; revised manuscript received 28 November 2016; published 31 January 2017; publisher error corrected 8 February 2017)

In the analysis of the robustness of multiplex networks, it is commonly assumed that a node is functioning only if its interdependent nodes are simultaneously functioning. According to this model, a multiplex network becomes more and more fragile as the number of layers increases. In this respect, the addition of a new layer of interdependent nodes to a preexisting multiplex network will never improve its robustness. Whereas such a model seems appropriate to understand the effect of interdependencies in the simplest scenario of a network composed of only two layers, it may seem unsuitable to characterize the robustness of real systems formed by multiple network layers. In fact, it seems unrealistic that a real system evolved, through the development of multiple layers of interactions, towards a fragile structure. In this paper, we introduce a model of percolation where the condition that makes a node functional is that the node is functioning in at least two of the layers of the network. The model reduces to the commonly adopted percolation model for multiplex networks when the number of layers equals two. For larger numbers of layers, however, the model describes a scenario where the addition of new layers boosts the robustness of the system by creating redundant interdependencies among layers. We prove this fact thanks to the development of a message-passing theory that is able to characterize the model in both synthetic and real-world multiplex graphs.

DOI: 10.1103/PhysRevX.7.011013

\section{INTRODUCTION}

Multilayer networks [1-5] are emerging as a powerful paradigm for describing complex systems characterized by the coexistence of different types of interactions or coupling among different types of networks. These networks represent an appropriate descriptive model for real networked systems in disparate contexts, such as social $[6,7]$, technological [8-10], and biological systems [11-13], just to mention a few. For example, global infrastructures are formed by several interdependent networks, such as power grids, water supply networks, and communication systems [4]. Cell function and/or malfunction (yielding diseases) cannot be understood if the information on the different nature of the interactions forming the interactome (protein-protein interactions, signaling, regulation) is not integrated in a general multilayer scenario [11]. Similarly, the complexity of the brain is encoded in the different nature of the interactions existing at the functional and the structural levels $[12,13]$.

Published by the American Physical Society under the terms of the Creative Commons Attribution 4.0 International license. Further distribution of this work must maintain attribution to the author(s) and the published article's title, journal citation, and DOI.
Subject Areas: Complex Systems

General multilayer networks, as well as multiplex networks [1-3,14-16], are composed of nodes belonging to different layers and links connecting nodes within and across layers. In general multilayer networks, there are no restrictions in the way the links across different layers can be placed. In multiplex networks, however, the nodes of each pair of layers are mapped one to one, and the links across different layers can only be present among corresponding nodes. Therefore, multiplex networks are a specific case of multilayer networks with a well-defined structure. In multilayer networks, and multiplex networks as well, nodes belonging to different layers are often interdependent on each other, in the sense that a failure of one node might cause the failure of a node in another layer. For example, in a global infrastructure network, a power plant might be interdependent on a node of the communication system that is controlling its function [4].

Percolation models are generally used as proxies to quantify the robustness of networked systems under local failures, by monitoring how the connectedness at the macroscopic level changes as a function of the amount of microscopic damage of the individual elements of the system. Although different percolation models can be suitably defined and studied on multilayer networks (e.g., $k$-core percolation [17], weak percolation [18], and 
bond percolation [19]), here we focus our attention on the case of the so-called site-percolation model, where the units that can be potentially damaged are the nodes of the network and the order parameter of interest is the macroscopic connectedness of the system. When interdependencies are taken into account, the resulting percolation theory [4,20-29] provides a general framework to characterize the dramatic avalanches of failure that can affect multilayer networks. A large volume of publications have pointed out that, according to this model, a multilayer network is much more fragile than each of the various network layers taken in isolation [4,20-25]. In particular, the overall fragility of the system increases as the number of layers increases [23,26-29]. Such a feature has an intuitive explanation. In this percolation model, a node is damaged if at least one of its interdependent nodes is damaged. As the number of layers increases, the probability of individual failures grows, thus making the system more fragile. However, this scenario leads to the following conundrum: If the fragility of a system is increased by the number of layers of interactions, why are there so many real systems that display multiple layers of interactions? Furthermore, the addition of new layers of interactions in a preexisting multilayer network generally comes at a cost, so it does not seem reasonable to spend resources just to make the system less robust. For instance, according to a recent study focusing on diffusion dynamics on multimodal transportation networks [9], the presence of multiple interconnected modes of transportation makes the system more navigable and more robust than its individual layers considered in isolation. However, the aforementioned conundrum has not been fully addressed in terms of purely topological properties. The purpose of the current paper is to provide a potential explanation by introducing a suitable model for percolation in multiplex networks composed of multiple interacting layers. In the model, we assume that a node is damaged only if all of its interdependent nodes are simultaneously damaged. The model is perfectly equivalent to the one currently in use when the number of layers equals two. Additional layers, however, provide the system with redundant interdependencies, generating backup mechanisms against the failure of the system, and thus making it more robust.

Percolation in the absence of interdependencies has been studied in multilayer networks, finding a "complementary" and synergistic role of different layers [13,19,30-32]. Here, these effects are observed despite the presence of interdependencies. We provide a comprehensive study of percolation in the presence of redundant interdependencies thanks to the development of an exhaustive messagepassing theory [20,33-36] (also known as the cavity method). We build on recent advances obtained in the "standard" percolation theory for multilayer networks $[26,37-40]$ to propose a theory that is valid for arbitrary systems (thus also including overlap among layers [7,16]), as long as the network structure is locally treelike. This limitation is common to all message-passing approaches for studying critical phenomena on networks. Corrections have recently been proposed [41] on single networks to improve the performance of message-passing theory, and similar approximations valid for loopy multilayer networks might be envisaged in the future. We remark that our model represents a starting point to address an obvious, yet neglected feature that makes percolation more realistic as a model to study the robustness of real multilayer networks. Eventual modifications and/or the addition of further ingredients to the model presented here may still be necessary to deal with specific scenarios to make the model even more realistic.

\section{REDUNDANT PERCOLATION MODEL ON MULTIPLEX NETWORKS}

A multilayer network structure is not equivalent to a large single network. As a network is ultimately a way to encode information about a complex system, distinguishing between different types of links and nodes may significantly alter the characteristics of the structure and the dynamical behavior of the system. This fact is particularly evident when we associate a different role to links within each layer (intralinks) and links across different layers (interlinks). For example, when describing the diffusion in multilayer networks [42], we might reasonably assume that the diffusion constant along intralinks is different from the diffusion constant among interlinks, and this significantly changes the typical time scale of the dynamics. Similar considerations are also valid for spreading processes in multilayer networks [43]. In the context of percolation theory, attributing a different role to interlinks and intralinks can yield a scenario significantly different from percolation in single layers. Specifically, if interlinks describe interdependencies between the nodes, the percolation transition becomes discontinuous and hybrid $[4,21,25]$, and close to the percolation transition, the multilayer network is affected by large avalanches of cascading failures. In this case, we refer to the multilayer network as a set of interdependent networks. Therefore, "interdependent networks" is a term that specifically refers to the response of the system to the damage of the nodes more than to the actual structure of the multilayer network.

Depending on the type and number of interlayer connections present in the system, different classes of multilayer networks $[1-3,14]$ can be considered. Here, we deal with one of the simplest classes characterized by the fact that every node is connected to one and only one node in each of the other layers. These systems are generally named "multiplex networks" [1-3,14-16]. Very often, the linked nodes across different layers (also called replica nodes) actually describe different realization of the same node. For example, in the London transportation network, replica nodes could represent the Oxford Circus tube station and the Oxford Circus bus station. However, 
multiplex networks can also be used to indicate the scenario where nodes belonging to the various layers represent physically distinct units as long as the nodes of the different layers are mapped one to one and the cross-layer links are placed among all the corresponding (replica) nodes and nowhere else. For instance, multiplex networks can be used to model interdependent infrastructures when the interdependencies occur exclusively between replica nodes. Systems of this type are interdependent multiplex networks $[4,21,22,44]$, also named one-to-one interdependent networks.

We consider a multiplex network composed of $M$ layers $G_{\alpha}$, with $\alpha=1,2, \ldots, M$. We indicate the set of all layers as $\vec{G}=\left(G_{1}, G_{2}, \ldots, G_{M}\right)$. Every layer contains $N$ nodes. Exactly one node with the same label appears in every individual layer. Nodes in the various layers sharing a common label are called replica nodes, and they are considered interdependent on each other. Nodes in the network are identified by a pair of labels $(i, \alpha)$, with $i=1,2, \ldots, N$ and $\alpha=1,2, \ldots, M$, the first one indicating the index of the node and the second one standing for the index of the layer. For every node label $i$, the set of replica nodes is given by the $M$ nodes corresponding to pairs of labels $(i, \alpha)$ with $\alpha=1,2, \ldots, M$ (see Fig. 1 ). When at least two replica nodes $(i, \alpha)$ and $\left(i, \alpha^{\prime}\right)$ are connected to two corresponding replica nodes $(j, \alpha)$ and $\left(j, \alpha^{\prime}\right)$, we say that the multiplex network displays link overlap.

Given a multiplex network as described above, we consider a percolation model where some of the nodes are initially damaged. We assume that interlinks represent

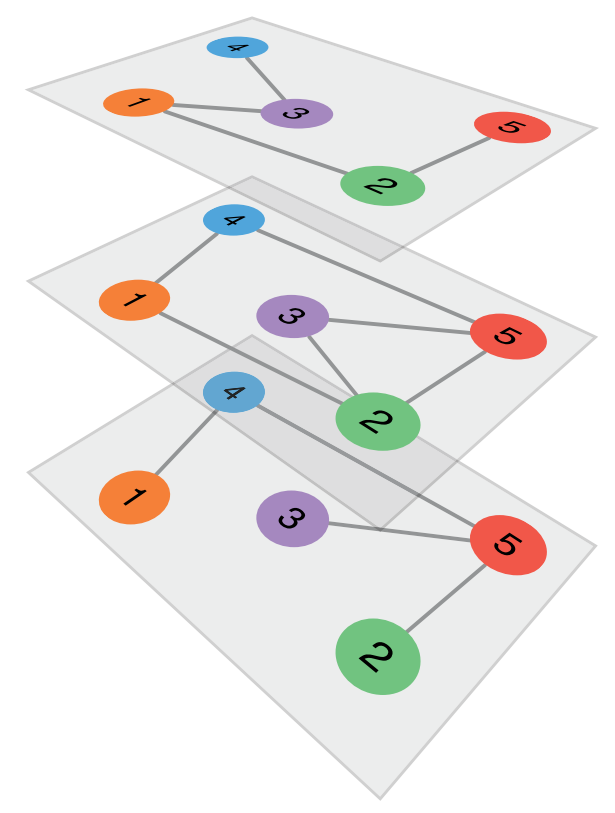

FIG. 1. A multiplex network with $M=3$ layers and $N=5$ nodes is shown. Every node $i$ has $M=3$ interdependent replica nodes $(i, \alpha)$ with $\alpha=1,2,3$. In this figure, triplets of replica nodes are also identified by their color. interdependencies among replica nodes, but we consider the case in which such interdependencies are redundant; i.e., every node can be active only if at least one of its interdependent nodes is also active. We refer to this model as the "redundant percolation model." As an order parameter for the model, we define the so-called redundant mutually connected giant component (RMCGC). The nodes that belong to the RMCGC can be found by following the algorithm:

(i) The giant component of each layer $\alpha$ is determined by evaluating the effect of the damaged nodes in each single layer.

(ii) Every replica node that has no other replica node in the giant component of its proper layer is removed from the network and considered damaged.

(iii) If no new damaged nodes are found in step (ii), then the algorithm stops; otherwise, it proceeds, starting again from step (i).

The set of replica nodes that are not damaged when the algorithm stops belongs to the RMCGC.

The main difference with the percolation model introduced in Ref. [4] and the consequent definition of the mutually connected giant component (MCGC) is that step (ii) must be substituted with the following: "Every replica node that has at least a single replica node not in the giant component of its proper layer is removed from the network and considered damaged; i.e., if a replica node is damaged, all of its interdependent replica nodes are damaged" [4,20-23,26-28]. In particular, the RMCGC and the MCGC are the same for $M=2$ layers, but they differ as long as the number of layers $M>2$. In the latter case, the RMCGC naturally introduces the notion of redundancy or complementarity among interdependent nodes.

Note that the choice of considering just two operating layers, i.e., assuming that a replica node can remain functional as long as there is at least another interdependent replica node that is also functional, is a simplification. In a real scenario, redundant interdependencies can include more than two operating layers or even a different number of operating layers for each node.

As a proof of concept to demonstrate the difference between the notions of MCGC and RMCGC, in Fig. 2 we present results of numerical simulations for the two percolation models applied to the air transportation network within the US [39]. We remark that this analysis represents only a starting point to illustrate the key impact of the setting of redundant interdependency. Airports are the nodes in network. Two airports are connected if at least one flight is connecting them. Layers correspond to flights operated by the three major carriers in the US: American Airlines, Delta, and United. In this system, having a connected component that is shared by at least two carriers could be important for several reasons. For example, competition on similar itineraries may favor a market for 


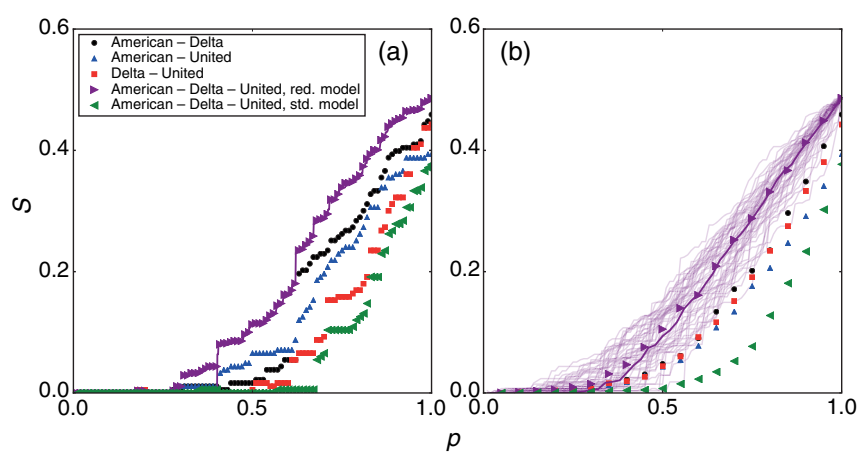

FIG. 2. Percolation transition in the US air transportation network [39]. We consider only US domestic flights operated in January 2014 by the three major carriers in the US (American Airlines, Delta, and United), and we construct a multiplex network where airports are nodes, and connections on the layers are determined by the existence of at least one flight operated by a given carrier between the two locations. The number of nodes in the network is $N=183$. Please note that some of the nodes appear as connected only in one layer; therefore, the relative size of the largest cluster is always smaller than one. (a) We consider a single realization of the random damage by assigning to every replica node $(i, \alpha)$ a random number extracted uniformly in the interval $[0,1]$. Replica nodes are considered damaged if their associated random number is smaller than $p$. Note that the same exact configuration of random damage is considered in all cases. In the percolation diagram, we consider the size of the MCGC and RMCGC as a function of $p$. Large symbols are results of numerical simulations, whereas the tick line is obtained from the solution of our mathematical framework [system of Eqs. (1)-(4)]. In particular, we consider the three possible multiplex networks composed of only two layers: American-Delta (black circles), American-United (blue triangles), and Delta-United (red squares). Diagrams obtained by considering the system as composed of all three layers are represented as green triangles for the model of Ref. [4] and as purple triangles for the redundant model. (b) Same as in panel (a) but for average values over 100 independent realizations of the random damage. Purple thin lines stand for the 100 independent realizations of random damage considered in the case of the redundant model. As is apparent from the figure, the average does not capture the behavior of single instances of disorder well, and fluctuations are rather large for a wide range of possible values of $p$.

plane tickets that is more fair than the one that would be present in the case of a monopoly by a single carrier. If only two layers are considered, MCGC and RMCGC coincide and both metrics indicate the relative size of the system where no monopoly is present. The addition of a third layer in the system should have beneficial effects for the system by increasing the size of the system where a monopoly is absent. This scenario is described by the redundant percolation model. Beneficial effects of the complementarity among routes offered by the various carriers are not only visible when the system is fully functional (i.e., parameter $p=1$ ) but also when a relatively large fraction of airports are considered to be removed from the system (approximately for $p \geq 0.5$ ). The percolation model introduced in Ref. [4] instead describes a much more restrictive situation, where the largest cluster is formed by airports that are connected simultaneously by all three carriers. The considerations reported above are valid for results for both a single instance of the percolation model [Fig. 2(a)] and for average values over multiple instances of the model [Fig. 2(b)].

As is clear from Fig. 2, our theory is able to reproduce with high accuracy the results of numerical simulations. The next sections are devoted to the description of a complete mathematical framework that allows for the description of the redundant percolation model. We stress that the framework is devised for arbitrary topologies and can therefore also be applied safely to real multilayer networks as long as their structures are sufficiently compatible with the locally treelike approximation.

\section{MESSAGE-PASSING ALGORITHM}

We assume that interactions within each layer $\alpha$ are described by elements $a_{i j}^{[\alpha]}$ of the adjacency matrix of the layer, indicating whether the replica nodes $(i, \alpha)$ and $(j, \alpha)$ are connected $\left(a_{i j}^{[\alpha]}=1\right)$ or not $\left(a_{i j}^{[\alpha]}=0\right)$ in layer $\alpha$. Additionally, we consider a specific realization of the initial damage to the replica nodes indicated by the set $\left\{s_{i \alpha}\right\}$. The generic element $s_{i \alpha}=0$ indicates that the replica node $(i, \alpha)$ has been initially damaged, whereas $s_{i \alpha}=1$ indicates that the replica node $(i, \alpha)$ has not been initially damaged. Under these conditions, as long as the multiplex network is locally treelike, the following message-passing algorithm identifies the replica nodes that are in the RMCGC.

Each node $i$ sends to a neighbor $j$ a set of messages $n_{i \rightarrow j}^{[\alpha]}$ in every layer $\alpha$, where node $i$ is connected to node $j$, i.e., with $a_{i j}^{[\alpha]}=1$. These messages indicate whether $\left(n_{i \rightarrow j}^{[\alpha]}=1\right)$ or not $\left(n_{i \rightarrow j}^{[\alpha]}=0\right)$ node $i$ connects node $j$ to the RMCGC with links belonging to layer $\alpha$. The message $n_{i \rightarrow j}^{[\alpha]}=1$ if and only if all of the following conditions are met:

(a) node $i$ is connected to node $j$ in layer $\alpha$, and neither node $(i, \alpha)$ nor node $(j, \alpha)$ is damaged, i.e., $s_{i \alpha}=s_{j \alpha}=a_{i j}^{[\alpha]}=1$;

(b) node $i$ is connected to the RMCGC through at least one node $\ell \neq j$ in layer $\alpha$;

(c) node $i$ belongs to the RMCGC, assuming that node $j$ also belongs to the RMCGC. This condition is satisfied if and only if, assuming that node $j$ belongs to the RMCGC, node $i$ is connected in at least two layers to the RMCGC.

If the previous conditions are not simultaneously met, then $n_{i \rightarrow j}^{[\alpha]}=0$. Put together, the former conditions lead to 
$n_{i \rightarrow j}^{[\alpha]}=\theta\left(v_{i \rightarrow j}, 2\right) a_{i j}^{[\alpha]} s_{j \alpha} s_{i \alpha}\left[1-\prod_{\ell \in N_{\alpha}(i) \backslash j}\left(1-n_{\ell \rightarrow i}^{[\alpha]}\right)\right]$.

Here, $N_{\alpha}(i)$ indicates the set of nodes that are neighbors of node $i$ in layer $\alpha$. The term $1-\prod_{\ell \in N_{\alpha}(i) \backslash j}\left(1-n_{\ell \rightarrow i}^{[\alpha]}\right)$ will therefore equal one if at least one message is arriving at node $i$ from a neighboring node $\ell \neq j$, while it will be equal to zero otherwise. Note that $\theta\left(v_{i \rightarrow j}, 2\right)=1$ for $v_{i \rightarrow j} \geq 2$ and $\theta\left(v_{i \rightarrow j}, 2\right)=0$ otherwise. Here, $v_{i \rightarrow j}$ indicates in how many layers node $i$ is connected to the RMCGC, assuming that node $j$ also belongs to the RMCGC, i.e.,

$$
\begin{aligned}
v_{i \rightarrow j}= & \sum_{\alpha=1}^{M}\left[s_{i \alpha}\left(1-\prod_{\ell \in N_{\alpha}(i) \backslash j}\left(1-n_{\ell \rightarrow i}^{[\alpha]}\right)\right)\right. \\
& \left.+s_{i \alpha} s_{j \alpha} a_{i j}^{[\alpha]} \prod_{\ell \in N_{\alpha}(i) \backslash j}\left(1-n_{\ell \rightarrow i}^{[\alpha]}\right)\right] .
\end{aligned}
$$

Therefore, $v_{i \rightarrow j}$ indicates the number of initially undamaged replica nodes $(i, \alpha)$ that either receive at least one positive message from nodes $\ell \in N_{\alpha}(i) \backslash j$ or are connected to the undamaged replica nodes $(j, \alpha)$. Finally, the replica node $(i, \alpha)$ belongs to the RMCGC if (i) it is not damaged, (ii) it is connected to the RMCGC in layer $\alpha$, and (iii) it receives at least one other positive message in a layer, $\alpha^{\prime} \neq \alpha$. These conditions are summarized by

$$
\begin{aligned}
\sigma_{i \alpha}= & s_{i \alpha}\left(1-\prod_{\ell \in N_{\alpha}(i)}\left(1-n_{\ell \rightarrow i}^{[\alpha]}\right)\right) \\
& \times\left\{1-\prod_{\alpha^{\prime} \neq \alpha}\left[1-s_{i \alpha^{\prime}}+s_{i \alpha^{\prime}} \prod_{\ell \in N_{\alpha^{\prime}}(i)}\left(1-n_{\ell \rightarrow i}^{\left[\alpha^{\prime}\right]}\right)\right]\right\} .
\end{aligned}
$$

The average number $S$ of replica nodes belonging to the RMCGC is computed as

$$
S=\frac{1}{M N} \sum_{\alpha=1}^{M} \sum_{i=1}^{N} \sigma_{i \alpha}
$$

The system of Eqs. (1)-(4) represents a complete mathematical framework to estimate the average size of the RMCGC for a given network and a given initial configuration of damage. The solution can be obtained by first iterating Eqs. (1) and (2) to obtain the values of the messages $n_{i \rightarrow j}^{[\alpha]}$. Those values are then plugged into Eq. (3) to compute the values of the variables $s_{i \alpha}$, and finally, these variables are used in Eq. (4) to estimate the average size of the RMCGC. We stress that, being valid for a given network and for a given configuration of damage, the values of the variables $n_{i \rightarrow j}^{[\alpha]}$ and $s_{i \alpha}$ are either 0 or 1 . The variables $v_{i \rightarrow j}$ can assume instead integer values in the range $[0, M]$. The mathematical framework also works properly in the presence of edge overlap among layers. This is an important feature that can dramatically change the robustness properties of multilayer networks [26,37-40].

\section{MULTIPLEX NETWORKS WITHOUT LINK OVERLAP}

\section{A. General results}

\section{Simplification of the message-passing equations on a single realization of the initial damage}

In the absence of link overlap, a given pair of nodes $i$ and $j$ may be linked exclusively along a single layer $\alpha$. Nontrivial messages that are potentially different from zero will therefore exist only on a specific layer for every pair of connected nodes $i$ and $j$. It can easily be seen that the message-passing equations (1) and (2) reduce to

$$
\begin{aligned}
n_{i \rightarrow j}^{[\alpha]}= & s_{i \alpha} s_{j \alpha} a_{i j}^{[\alpha]}\left[1-\prod_{\ell \in N_{\alpha}(i) \backslash j}\left(1-n_{\ell \rightarrow i}^{[\alpha]}\right)\right] \\
& \times\left\{1-\prod_{\alpha^{\prime} \neq \alpha}\left[1-s_{i \alpha^{\prime}}+s_{i \alpha^{\prime}} \prod_{\ell \in N_{\alpha^{\prime}}(i)}\left(1-n_{\ell \rightarrow i}^{\left[\alpha^{\prime}\right]}\right)\right]\right\} .
\end{aligned}
$$

We further notice that, in this situation, the result of the message-passing algorithm does not change if we consider messages that depend exclusively on the state $s_{i \alpha}$ of the node $i$ that sends the message. Even if we drop the factor $s_{j \alpha}$ in Eq. (5), the message will still be allowed to propagate further at the next iteration step, if the replica node $(j, \alpha)$ is not initially damaged. Therefore, we can further simplify Eq. (5) and consider

$$
\begin{aligned}
n_{i \rightarrow j}^{[\alpha]}= & s_{i \alpha} a_{i j}^{[\alpha]}\left[1-\prod_{\ell \in N_{\alpha}(i) \backslash j}\left(1-n_{\ell \rightarrow i}^{[\alpha]}\right)\right] \\
& \times\left\{1-\prod_{\alpha^{\prime} \neq \alpha}\left[1-s_{i \alpha^{\prime}}+s_{i \alpha^{\prime}} \prod_{\ell \in N_{\alpha^{\prime}}(i)}\left(1-n_{\ell \rightarrow i}^{\left[\alpha^{\prime}\right]}\right)\right]\right\} .
\end{aligned}
$$

Equation (6) replaces Eqs. (1) and (2) in the case of a multiplex network without link overlap. The rest of the framework is identical, so Eqs. (3) and (4) remain unchanged.

\section{Message-passing equations for random realizations of the initial damage}

Equations (3), (4), and (6) determine the average size of the RMCGC in a multiplex network without link overlap for a given realization of the initial damage $\left\{s_{i \alpha}\right\}$. These equations can, however, be extended to make predictions in the case of a random realization of the initial damage when 
the replica nodes are damaged independently with probability $1-p$, i.e., such that the initial damage $\left\{s_{i \alpha}\right\}$ is a random configuration obeying the probability distribution

$$
\hat{\mathcal{P}}\left(\left\{s_{i \alpha}\right\}\right)=\prod_{i=1}^{N} \prod_{\alpha=1}^{M} p^{s_{i \alpha}}(1-p)^{1-s_{i \alpha}} .
$$

To this end, we denote the probability that node $i$ sends a positive message to node $j$ in layer $\alpha$ by $\hat{n}_{i \rightarrow j}^{[\alpha]}=\left\langle n_{i \rightarrow j}^{[\alpha]}\right\rangle$, and the probability that the replica node $(i, \alpha)$ belongs to the RMCGC by $\hat{\sigma}_{i \alpha}=\left\langle\sigma_{i \alpha}\right\rangle$. The message-passing algorithm determining the values of $\hat{n}_{i \rightarrow j}^{[\alpha]}$ and $\hat{\sigma}_{i \alpha}$ is given by

$$
\begin{aligned}
\hat{n}_{i \rightarrow j}^{[\alpha]}= & a_{i j}^{[\alpha]} p\left[1-\prod_{\ell \in N_{\alpha}(i) \backslash j}\left(1-\hat{n}_{\ell \rightarrow i}^{[\alpha]}\right)\right] \\
& \times\left\{1-\prod_{\alpha^{\prime} \neq \alpha}\left[1-p+p \prod_{\ell \in N_{\alpha^{\prime}}(i)}\left(1-\hat{n}_{\ell \rightarrow i}^{\left[\alpha^{\prime}\right]}\right)\right]\right\}, \\
\hat{\sigma}_{i \alpha}= & p\left(1-\prod_{\ell \in N_{\alpha}(i)}\left(1-\hat{n}_{\ell \rightarrow i}^{[\alpha]}\right)\right) \\
& \times\left\{1-\prod_{\alpha^{\prime} \neq \alpha}\left[1-p+p \prod_{\ell \in N_{\alpha^{\prime}}(i)}\left(1-\hat{n}_{\ell \rightarrow i}^{\left[\alpha^{\prime}\right]}\right)\right] .\right.
\end{aligned}
$$

This algorithm can be applied to a given network, and it provides the average number of replica nodes $S$ belonging to the RMCGC for a random realization of the initial damage obeying Eq. (7). Specifically, the value of $\hat{S}$ is related to $\hat{\sigma}_{i \alpha}$ by

$$
\hat{S}=\frac{1}{M N} \sum_{i=1}^{N} \sum_{\alpha=1}^{M} \hat{\sigma}_{i \alpha} .
$$

\section{Message-passing equations for random multiplex networks}

A multiplex network in which every layer is a sparse network generated according to the configuration model is a major example of a multiplex network without link overlap in the limit of large network sizes. It is therefore natural and important to characterize the RMCGC in this case. We assume that every network layer $G_{\alpha}$ is a random graph taken from the probability distribution

$$
\mathcal{P}^{[\alpha]}\left(G_{\alpha}\right)=\frac{1}{Z} \prod_{i=1}^{N} \delta\left(k_{i}^{[\alpha]}, \sum_{j=1}^{N} a_{i j}^{[\alpha]}\right)
$$

where $k_{i}^{[\alpha]}$ indicates the pre-imposed degree of node $i$ in layer $\alpha, \delta(x, y)=1$ if $x=y$ and $\delta(x, y)=0$ otherwise, and $Z$ is the normalization factor indicating the total number of networks in the ensemble. Averaging over the network ensemble allows us to translate the message-passing equations into simpler expressions for the characterization of the percolation transition.

Let us consider a random multiplex network obeying the probability of Eq. (10), and a random realization of the initial damage described by the probability of Eq. (7). The average message in layer $\alpha$, namely, $S_{\alpha}^{\prime}=\left\langle\hat{n}_{i \rightarrow j}^{\alpha} \mid a_{i j}^{[\alpha]}=1\right\rangle$, and the average number of replica nodes of layer $\alpha$ that are in the RMCGC, denoted by $S_{\alpha}=\left\langle\hat{\sigma}_{i, \alpha}\right\rangle$, obey the equations

$$
\begin{aligned}
S_{\alpha}= & p \sum_{\mathbf{k}} P(\mathbf{k})\left[1-\left(1-S_{\alpha}^{\prime}\right)^{\left.k^{[\alpha]}\right]}\right. \\
& \times\left\{1-\prod_{\alpha^{\prime} \neq \alpha}\left[1-p+p\left(1-S_{\alpha^{\prime}}^{\prime}\right)^{\left.k^{\left[\alpha^{\prime}\right]}\right]}\right],\right. \\
S_{\alpha}^{\prime}= & p \sum_{\mathbf{k}} \frac{k^{\alpha}}{\left\langle k^{\alpha}\right\rangle} P(\mathbf{k})\left[1-\left(1-S_{\alpha}^{\prime}\right)^{k^{[\alpha]}-1}\right] \\
& \times\left\{1-\prod_{\alpha^{\prime} \neq \alpha}\left[1-p+p\left(1-S_{\alpha^{\prime}}^{\prime}\right)^{\left.k^{\left[\alpha^{\prime}\right]}\right]}\right],\right.
\end{aligned}
$$

where $P(\mathbf{k})$ indicates the probability that a generic node $i$ has degrees $\mathbf{k}_{i}=\mathbf{k}$, i.e., $\left(k_{i}^{[1]}, k_{i}^{[2]}, \ldots, k_{i}^{[M]}\right)=$ $\left(k^{[1]}, k^{[2]}, \ldots, k^{[M]}\right)$.

If there are no correlations between the degrees of a node in different layers, the degree distribution $P(\mathbf{k})$ can be factorized as

$$
P(\mathbf{k})=\prod_{\alpha} P^{[\alpha]}\left(k^{[\alpha]}\right),
$$

where $P^{[\alpha]}(k)$ is the degree distribution in layer $\alpha$. In this case, Eqs. (11) can be expressed in terms of the generating function of the degree distribution in each layer. Specifically, we have

$$
\begin{aligned}
S_{\alpha}= & p\left[1-H_{0}^{[\alpha]}\left(1-S_{\alpha}^{\prime}\right)\right] \\
& \times\left\{1-\prod_{\alpha^{\prime} \neq \alpha}\left[1-p+p H_{0}\left(1-S_{\alpha^{\prime}}^{\prime}\right)\right]\right\}, \\
S_{\alpha}^{\prime}= & p\left[1-H_{1}^{[\alpha]}\left(1-S_{\alpha}^{\prime}\right)\right] \\
& \times\left\{1-\prod_{\alpha^{\prime} \neq \alpha}\left[1-p+p H_{0}^{\left[\alpha^{\prime}\right]}\left(1-S_{\alpha^{\prime}}^{\prime}\right)\right]\right\},
\end{aligned}
$$

where the generating functions $H_{0}^{[\alpha]}(z)$ and $H_{1}^{[\alpha]}(z)$ of the degree distribution $P^{[\alpha]}(k)$ of layer $\alpha$ are given by

$$
\begin{aligned}
& H_{0}^{[\alpha]}(x)=\sum_{k} P^{[\alpha]}(k) x^{k}, \\
& H_{1}^{[\alpha]}(x)=\sum_{k} \frac{k}{\left\langle k^{[\alpha]}\right\rangle} P^{[\alpha]}(k) x^{k-1} .
\end{aligned}
$$


Finally, the average number $S$ of replica nodes in the RMCGC is given by

$$
S=\frac{1}{M} \sum_{\alpha} S_{\alpha}
$$

If we consider the case of equally distributed Poisson layers with average degree $z$, we have that Eq. (12) is

$$
P^{[\alpha]}(k)=\frac{1}{k !} z^{k} e^{-z}
$$

for every layer $\alpha=1,2, \ldots, M$. Then, using Eqs. (13), one can show that $S_{\alpha}^{\prime}=S_{\alpha}=S, \forall \alpha$, and $S$ is determined by the equation

$$
S=p\left(1-e^{-z S}\right)\left\{1-\left[1-p+p e^{-z S}\right]^{M-1}\right\} .
$$

This equation always has the trivial solution $S=0$. In addition, a nontrivial solution $S>0$ indicating the presence of the RMCGC emerges at a hybrid discontinuous transition characterized by a square root singularity, on a line of points $p=p_{c}(z)$, determined by the equations

$$
\begin{aligned}
h_{z, p}\left(S_{c}\right) & =0, \\
\left.\frac{d h_{z, p}(S)}{d S}\right|_{S=S_{c}} & =0,
\end{aligned}
$$

where

$$
h_{z, p}(S)=S-p\left(1-e^{-z S}\right)\left\{1-\left[1-p+p e^{-z S}\right]^{M-1}\right\}=0 .
$$

For $p>p_{c}$, there is a RMCGC; for $p \leq p_{c}$, there is no RMCGC. The entity of the discontinuous jump at $p=p_{c}$ in the fraction $S$ of replica nodes in the RMCGC is given by $S=S_{c}$. The percolation threshold $p_{c}$ as a function of the average degree $z$ of the network is plotted in Fig. 3 for $M=2,3,4,5$. It is shown that as the number of layers $M$ increases, the percolation threshold decreases for every value of the average degree $z$. Also, the discontinuous jump $S_{c}$ decreases as the number $M$ of layers increases, for every given value of the average degree $z$ (see Fig. 4). Therefore, as the number of layers increases, the multilayer networks becomes more robust.

\section{B. Comparison between the RMCGC and the MCGC}

In this section, we compare the robustness of multiplex networks in the presence of ordinary interdependencies and in the presence of redundant interdependencies. As a concrete example, we consider the case of a multiplex network with $M$ Poisson layers, each layer having the same average degree $z$. In this case, the fraction $S$ of replica

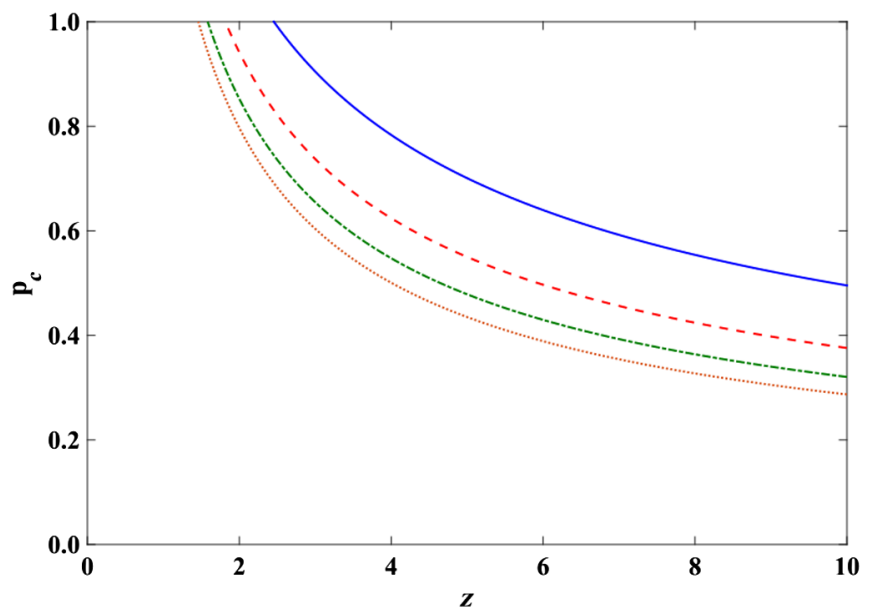

FIG. 3. The percolation threshold $p_{c}$ is plotted versus the average degree $z$ of each layer for Poisson multiplex networks with $M=2,3,4,5$ layers indicated, respectively, with blue solid, red dashed, green dot-dashed, and orange dotted lines.

nodes in the RMCGC is given by the solution of Eqs. (17), while the fraction of replica nodes in the MCGC is given by [45]

$$
S=\tilde{p}\left(1-e^{-z S}\right)^{M}
$$

In Eq. (20), it is assumed that every replica node $(i, \alpha)$ of a given node $i$ is damaged simultaneously (with probability $\tilde{f}=1-\tilde{p}$ ). On the contrary, in the presence of redundant interdependencies, it is natural to assume that the initial damage is inflicted on each replica node independently (with probability $f=1-p$ ). Therefore, in order to compare the robustness of the multiplex

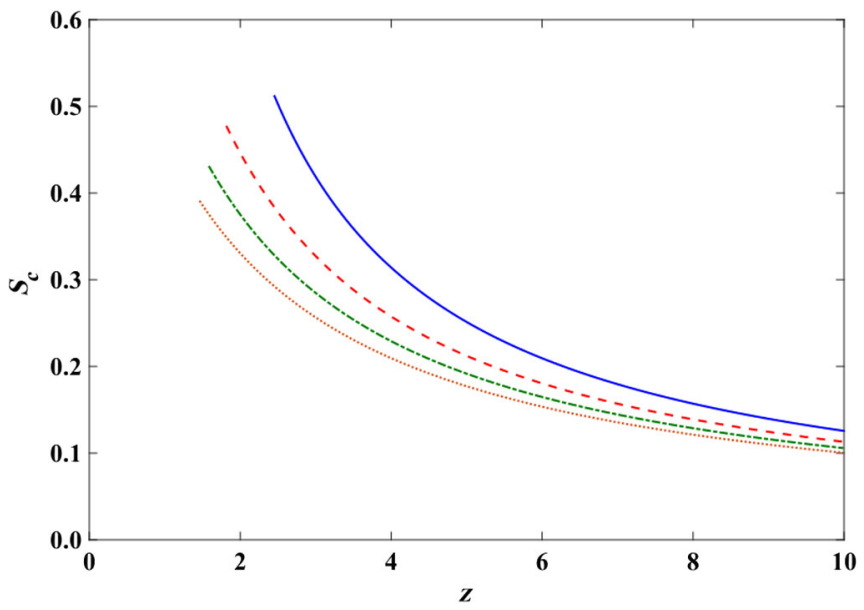

FIG. 4. The discontinuous jump $S_{c}=S\left(p_{c}\right)$ of the RMCGC at the percolation threshold $p=p_{c}$ is plotted versus the average degree $z$ of each layer for Poisson multiplex networks with $M=2,3,4,5$ layers indicated, respectively, with blue solid, red dashed, green dot-dashed, and orange dotted lines. 
networks in the presence and in the absence of redundant interdependencies, we set $p=\tilde{p}=1$ (i.e., replica nodes are not initially damaged) and compare the critical value of the average degree $z=z^{\star}$ at which the percolation transition occurs, respectively, for the RMCGC and for the MCGC. Additionally, we also characterize the size $S=S^{\star}$ of the jump in the size of the RMCGC and the MCGC at the percolation transition. In Fig. 5, we display the values of $z^{\star}$ and $S^{\star}$ as a function of the number of layers $M$ for the RMCGC and the MCGC. For $M=2$, the two models give the same results, as they are identical. For $M>2$, differences arise. In the presence of redundant interdependencies, multiplex networks become increasingly more robust as the number $M$ of layers increases. This phenomenon is apparent from the fact that the RMCGC emerges for multiplex networks with an average degree of their layers $z^{\star}$, which decreases as the number of layers $M$ increases. On the contrary, in ordinary percolation, the value of $z^{\star}$ for the emergence of the MCGC is an increasing function of $M$. Additionally, the size of the discontinuous jumps $S^{\star}$ at the transition point decreases with $M$ for the RMCGC, while the size increases with $M$ for the MCGC, showing that the avalanches of failures have a reduced size for the RMCGC. We expect that the beneficial effect of the addition of new layers will also extend to the scenario in which the number of operating layers necessary for a node to be functional is assumed to be lager than two. Also, we believe that the same conclusion will apply to more general multilayer network structures where nodes have different numbers of interlinks (redundant interdependencies), such as the topologies considered in Ref. [28], as long as the number of
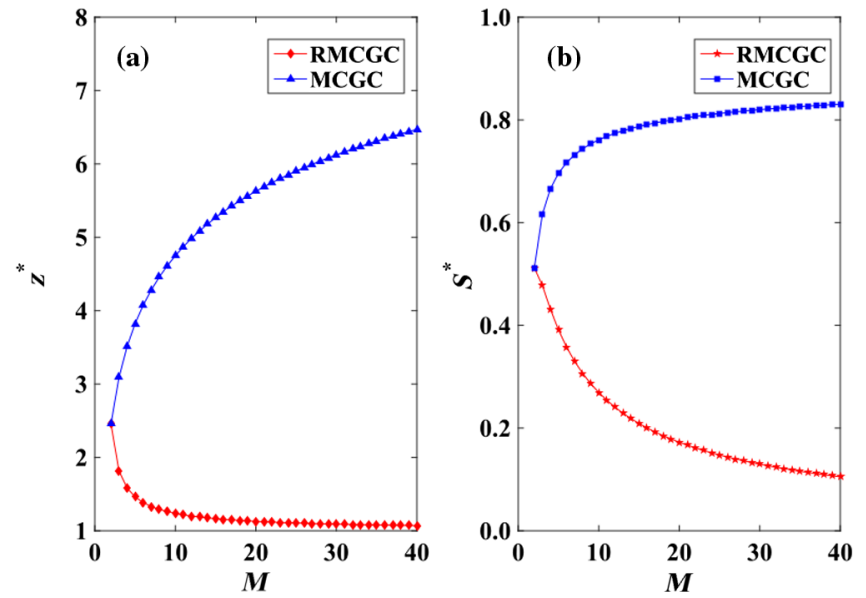

FIG. 5. Comparison between the MCGC and the RMCGC models in Poisson multiplex networks. (a) Critical value $z^{\star}$ of the average degree as a function of the number of network layers $M$. Results for the RMCGC model are displayed as red diamonds. Results for the MCGC model are denoted by blue triangles. (b) Height of the jump $S^{\star}$ at the transition point as a function of the number of network layers. operating layers necessary for a node to be functional is the same for every node.

\section{Comparison with numerical simulations}

In this section, we compare the results obtained with Eqs. (1)-(4) on a single instance of damage with the predictions of the message-passing algorithm described in Eq. (13) characterizing the size $S$ of the RMCGC in an ensemble of networks. Specifically, we consider the case of a multilayer network with $M=3$ Poisson layers with the same average degree $z$. In order to draw the percolation diagram for single instances of initial damage as a function of the probability of damage $1-p$, we associate each replica node $(i, \alpha)$ with a random variable $r_{i \alpha}$ drawn from a uniform distribution, and we set

$$
s_{i \alpha}=\left\{\begin{array}{lll}
1 & \text { if } & r_{i \alpha} \leq p \\
0 & \text { if } & r_{i \alpha}>p
\end{array}\right.
$$

Figure 6 displays the comparison between the two approaches, showing an almost perfect agreement between them. Additionally, in Fig. 7, we compare simulation results averaged over several realizations of the initial damage and several instances of the multiplex network model with the theoretical predictions given by the numerical solution of Eqs. (11)-(17), obtaining a very good agreement.

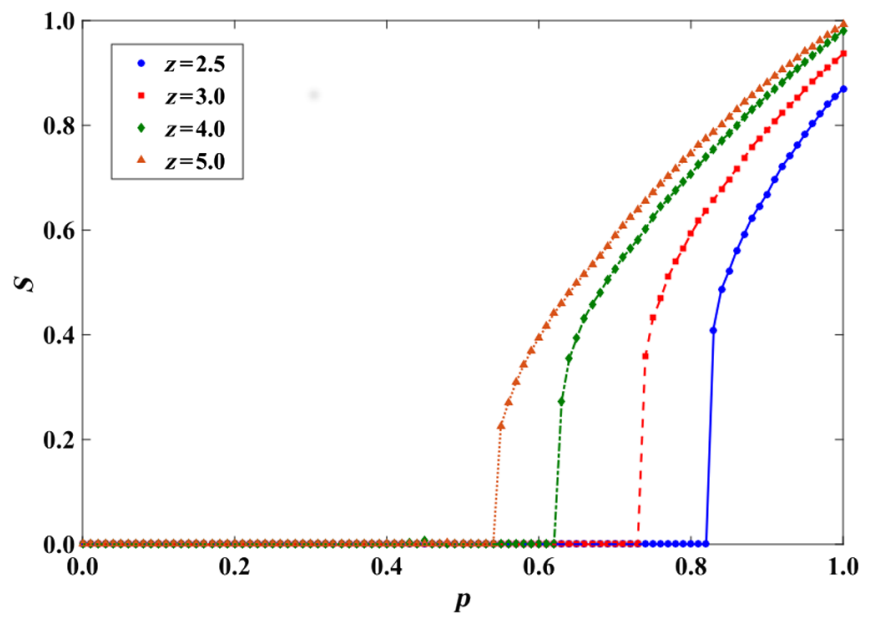

FIG. 6. Comparison between simulation results of the RMCGC for a multiplex network with $M=3$ Poisson layers with average degree $z$ and no link overlap, and the messagepassing results over single-network realization and given configuration damage. We consider different values of the average degree $z=2.5,3.0,4.0,5.0$. Points indicate results of numerical simulations: blue circles $(z=2.5)$, red squares $(z=3.0)$, green diamonds $(z=4.0)$, and orange triangles $(z=5.0)$. Message-passing predictions are denoted by lines with the same color scheme used for numerical simulations. Simulation results are performed on a single instance of a multiplex network with $N=10^{4}$ nodes. 


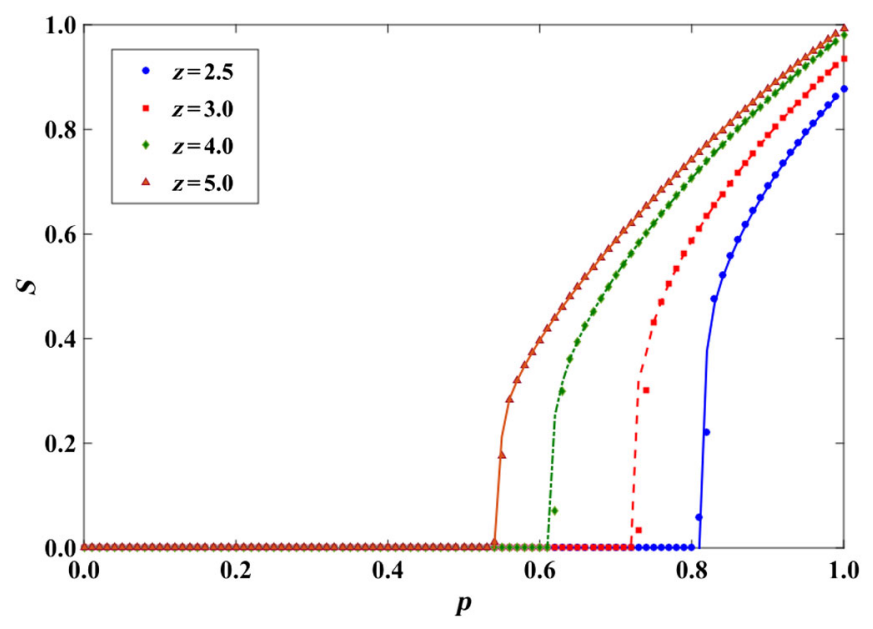

FIG. 7. Same as in Fig. 6, but for averages over 20 instances of the multiplex network model and configurations of random initial damage.

\section{MULTIPLEX NETWORKS WITH LINK OVERLAP}

\section{A. Link overlap, multilinks, and multidegree}

In isolated networks, two nodes can be either connected or not connected. In multiplex networks, instead, the complexity of the structure greatly increases as the ways in which a generic pair of nodes can be connected is given by $2^{M}$ possibilities. A very convenient way of accounting for all the possibilities with a compact notation is to use the notion of multilink among pairs of nodes [7,16]. Multilinks $\vec{m}=\left(m^{[1]}, m^{[2]}, \ldots, m^{[M]}\right)$, with $m^{[\alpha]}=0,1$, describe any of the possible patterns of connections between pairs of nodes in a multilayer network with $M$ layers. Specifically, $m^{[\alpha]}=1$ indicates that a connection exists in layer $\alpha$, whereas $m^{[\alpha]}=0$ indicates that the connection in layer $\alpha$ does not exist. In particular, we can say that, in a multiplex network with $M$ layers, two nodes $i$ and $j$ are connected by the multilink

$$
\vec{m}_{i j}=\left(a_{i j}^{[1]}, a_{i j}^{[2]}, \ldots, a_{i j}^{[M]}\right) .
$$

In order to distinguish the case in which two nodes are not connected in any layer with the case in which at least one layer of the nodes are connected, we distinguish between the trivial multilink $\vec{m}=\overrightarrow{0}$ and the nontrivial multilinks $\vec{m} \neq \overrightarrow{0}$. The trivial multilink $\vec{m}=\overrightarrow{0}$ indicates the absence of any sort of link between the two nodes.

Using the concept of multilinks, one can define multiadjacency matrices $\mathbf{A}^{\vec{m}}$ whose element $A_{i j}^{\vec{m}}$ indicates whether $\left(A_{i j}^{\vec{m}}=1\right)$ or not $\left(A_{i j}^{\vec{m}}=0\right)$ a node $i$ is connected to node $j$ by a multilink $\vec{m}$. The matrix elements $A_{i j}^{\vec{m}}$ of the multiadjacency matrix $\mathbf{A}^{\vec{m}}$ are given by

$$
A_{i j}^{\vec{m}}=\prod_{\alpha=1}^{M} \delta\left(m^{[\alpha]}, a_{i j}^{[\alpha]}\right) .
$$

We note that multiadjacency matrices are essentially equivalent to rank-3 tensors such as those considered in Ref. [15] for multiplex networks and those generalized to the case of arbitrary multilayer networks in Ref. [14]. Using multiadjacency matrices, it is straightforward to define multidegrees [7,16]. The multidegree of node $i$ indicated as $k_{i}^{\vec{m}}$ is the sum of rows (or columns) of the multiadjacency matrix $\mathbf{A}^{\vec{m}}$, i.e.,

$$
k_{i}^{\vec{m}}=\sum_{j} A_{i j}^{\vec{m}}
$$

and it indicates how many multilinks $\vec{m}$ are incident to node $i$.

Using a multidegree sequence $\left\{k_{i}^{\vec{m}}\right\}$, it is possible to build multiplex network ensembles that generalize the configuration model. Thus, overlap of links is fully preserved by the randomization of the multiplex network. These ensembles are specified by the probability $\tilde{\mathcal{P}}(\vec{G})$ attributed to every multiplex network $\vec{G}$ of the ensembles, where $\tilde{\mathcal{P}}(\vec{G})$ is given by

$$
\tilde{\mathcal{P}}(\vec{G})=\frac{1}{\tilde{Z}} \prod_{i=1}^{N} \prod_{\vec{m} \neq 0} \delta\left(k_{i}^{\vec{m}}, \sum_{j=1}^{N} A_{i j}^{\vec{m}}\right),
$$

with the $\tilde{Z}$ normalization constant equal to the number of multiplex networks with given multidegree sequence.

\section{B. General discussion of the message-passing equations for the RMCGC}

Our goal here is to generalize the message-passing algorithm already given by Eqs. (1)-(4) for a generic single instance of a multiplex network and single realization of initial damage to the cases of (i) random multiplex networks with given multidegree sequence and/or (ii) random realizations of the initial damage. The extensions for both cases have already been considered for the case of multiplex networks without link overlap. In the presence of link overlap, however, a more complex formalism is needed. For two nodes $i$ and $j$ in fact, the messages $n_{i \rightarrow j}^{[\alpha]}$ given by Eq. (1) and sent from node $i$ to node $j$ over the different layers $\alpha=1,2, \ldots, M$ are correlated because they all depend on the value of the variable $v_{i \rightarrow j}$ given by Eq. (2). Such correlations require particular care when averaging the messages to treat the percolation transition for random initial damages. Similar technical challenges are also present in the treatment of the MCGC model where interdependencies are not redundant [26,37]. In the presence of redundant interdependencies, there is an additional 
precaution that needs to be taken. In fact, the messages $n_{i \rightarrow j}^{[\alpha]}$ are explicitly dependent on the state of all replicas $\left(j, \alpha^{\prime}\right)$ of node $j$. This state is indicated by the variables $\vec{s}_{j}=\left(s_{j 1}, s_{j 2}, \ldots, s_{j \alpha^{\prime}}, \ldots, s_{j M}\right)$, where $s_{j \alpha^{\prime}}$ specifies whether the replica node $\left(j, \alpha^{\prime}\right)$ is initially damaged or not. As a consequence of this property, when averaging over random realizations of initial damage, messagepassing equations are written in terms of the messages $\hat{\sigma}_{i \rightarrow j}^{\vec{m}_{i j}, \vec{n}}\left(\vec{s}_{j}\right)$ explicitly accounting for the probability that node $i$ is sending to node $j$ the set of messages $\vec{n}=\left(n_{i \rightarrow j}^{[1]}, n_{i \rightarrow j}^{[2]}, \ldots, n_{i \rightarrow j}^{[\alpha]}, \ldots, n_{i \rightarrow j}^{[M]}\right)$, given that node $j$ is in state $\vec{s}_{j}$ and nodes $i$ and $j$ are connected by a multilink $\vec{m}=\vec{m}_{i j}$. We have derived these equations for a general multiplex network with $M$ layers. However, the message-passing algorithm has a very long expression. To make the paper more readable, we decided to place the exact treatment of the general case in the standard model and consider here only the special case of ensembles of random multilayer networks with overlap. For these ensembles, in fact, the message-passing equations are written in terms of average messages sent between nodes with given multilinks $\vec{m}$, i.e., $S^{\vec{m}, \vec{n}}\left(\vec{s}_{j}\right)=\left\langle\hat{\sigma}_{i \rightarrow j}^{\vec{\sigma}_{i j}, \vec{n}^{\prime}}\left(\vec{s}_{j}\right) \mid \vec{m}_{i j}=\vec{m}\right\rangle$, and the equations greatly simplify. Two specific cases of multilayer network ensembles are discussed below, for the cases of $M=2$ and $M=3$ layers.

\section{Ensembles of multilayer network link overlap and $M=2$ layers}

In this case, every replica node is in the RMCGC if and only if its interdependent node in the other layer is also in the RMCGC. Therefore, the only messages that are different from zero are the messages $S^{\vec{m}, \vec{n}}\left(\vec{s}_{j}=(1,1)\right)$ sent to nodes $j$ in state $\vec{s}_{j}=(1,1)$. Specifically, we consider the case of a random multiplex network with Poisson multidegree distributions characterized by the averages

$$
\begin{aligned}
& \left\langle k^{(1,1)}\right\rangle=z_{2}, \\
& \left\langle k^{(0,1)}\right\rangle=\left\langle k^{(1,0)}\right\rangle=z_{1} .
\end{aligned}
$$

The messages $S^{\vec{m}, \vec{n}}\left(\vec{s}_{j}=(1,1)\right)$ only depend on the multiplicity of overlap of the multilinks $\vec{m}$ and $\vec{n}$ given, respectively, by

$$
\mu=\sum_{\alpha=1}^{M} m^{[\alpha]}, \quad \nu=\sum_{\alpha=1}^{M} n^{[\alpha]} .
$$

The fraction $S$ of replica nodes in the RMCGC is determined by the variables

$$
x_{\mu, \nu}=S^{\vec{m}, \vec{n}}\left(\vec{s}_{j}=(1,1)\right),
$$

where, for example, the value of $x_{2,2}$ indicates the probability that node $i$ sends a message $\vec{n}=(1,1)$ to its neighbor $j$ with $\vec{s}_{j}=(1,1)$ connected by a multilink $\vec{m}=(1,1)$.

The values of the variables $x_{\mu, \nu}$ and $S$ are determined by the following set of equations (see Ref. [46] for details):

$$
\begin{aligned}
x_{2,2} & =p^{2}\left[1-2 e^{-z_{1} x_{1,1}-z_{2}\left(x_{2,2}+x_{2,1}\right)}+e^{-2 z_{1} x_{1,1}-z_{2}\left(x_{2,2}+2 x_{2,1}\right)}\right], \\
x_{2,1} & =p^{2}\left[e^{-z_{1} x_{1,1}-z_{2}\left(x_{2,2}+x_{2,1}\right)}-e^{-2 z_{1} x_{1,1}-z_{2}\left(x_{2,2}+2 x_{2,1}\right)}\right], \\
S & =x_{1,1}=x_{2,2} .
\end{aligned}
$$

These equations are the same equations as those that determine the value of the MCGC as long as we make the substitution $p^{2} \rightarrow p[26,37]$, taking into account that the damage in each replica node is independent in the present model. Notably, in this case, the percolation phase transition is discontinuous and hybrid and is characterized by a square-root singularity for $p$ approaching the percolation threshold $p_{c}$ from above.

\section{Ensembles of multilayer network link overlap and $M=3$ layers}

We now consider the case of a random multiplex network with $M=3$ layers. The network has Poisson multidegree distributions and averages given by

$$
\begin{aligned}
& \left\langle k^{(1,1,1)}\right\rangle=z_{3}, \\
& \left\langle k^{(1,1,0)}\right\rangle=\left\langle k^{(1,0,1)}\right\rangle=\left\langle k^{(0,1,1)}\right\rangle=z_{2}, \\
& \left\langle k^{(1,0,0)}\right\rangle=\left\langle k^{(0,1,0)}\right\rangle=\left\langle k^{(0,0,1)}\right\rangle=z_{1} .
\end{aligned}
$$

In this case, the messages $S^{\vec{m}, \vec{n}}\left(\vec{s}_{j}\right)$ only depend on

$$
\mu=\sum_{\alpha=1}^{M} m^{[\alpha]}, \quad \nu=\sum_{\alpha=1}^{M} n^{[\alpha]}, \quad \xi=\sum_{\alpha=1}^{M} s_{j \alpha} m^{[\alpha]} .
$$

Therefore, the fraction of replica nodes in the RMCGC $S$ is determined by the variables

$$
x_{\mu, \nu}^{(\xi)}=S^{\vec{m}, \vec{n}}\left(\vec{s}_{j}\right)
$$

The equations that these variables need to satisfy can be described in a symbolic way by suitable diagrams (see Ref. [46] for details on how to read these diagrams). Diagrams that describe the equations to determine the value of all the variables $x_{\mu, \nu}^{(\xi)}$ are presented in Fig. 8. These equations read 


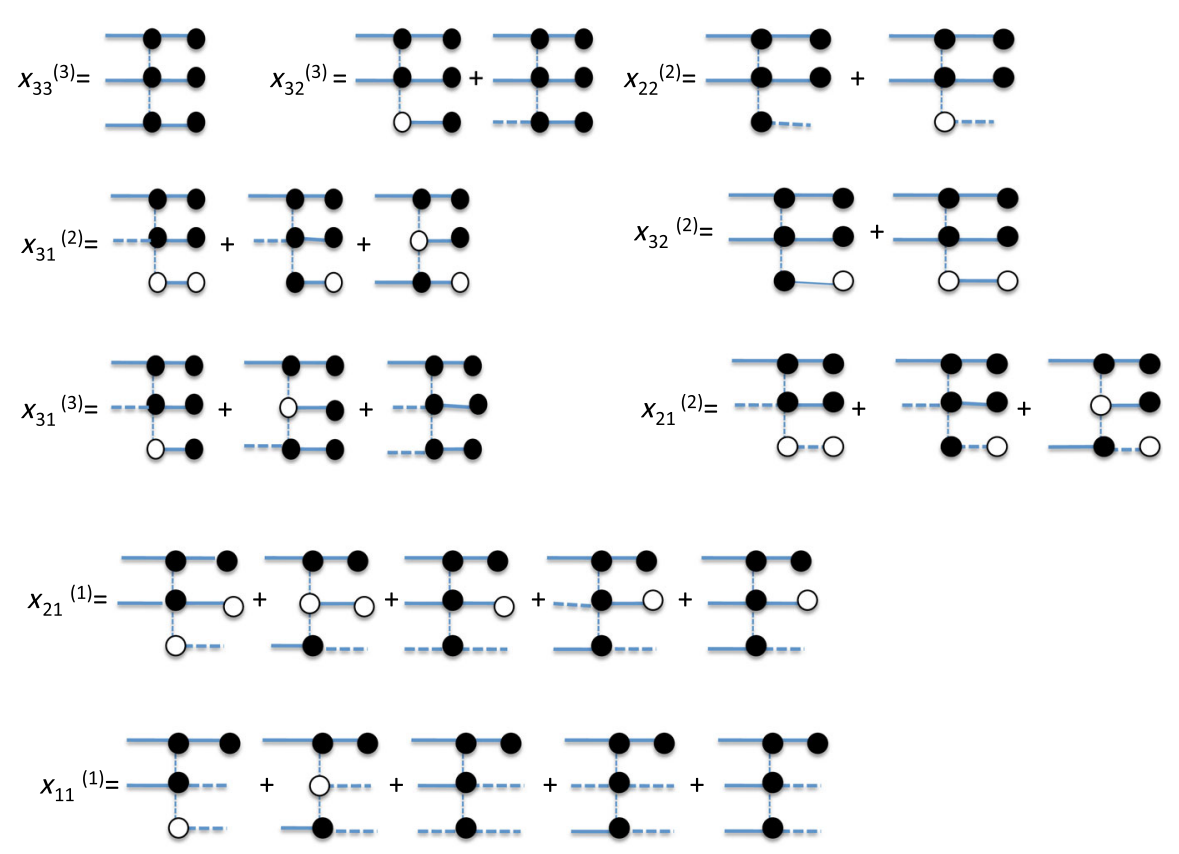

FIG. 8. Diagrams for Eqs. (33) determining $x_{\mu \nu}^{(\xi)}$ in the case of multiplex networks with three layers $(M=3)$ and Poisson multidegree distribution with $\left\langle k^{(1,1,1)}\right\rangle=z_{3},\left\langle k^{(1,1,0)}\right\rangle=\left\langle k^{(1,0,1)}\right\rangle=\left\langle k^{(0,1,1)}\right\rangle=z_{2}$ and $\left\langle k^{(1,0,0)}\right\rangle=\left\langle k^{(0,1,0)}\right\rangle=\left\langle k^{(0,0,1)}\right\rangle=z_{1}$.

$x_{3,3}^{(3)}=p^{3}\left[1-3 h_{1,3}+3 h_{2,3}-h_{3,3}\right]$,

$x_{3,2}^{(3)}=p^{2}(1-p)\left[1-2 h_{1,2}+h_{2,2}\right]+p^{3}\left[h_{1,3}-2 h_{2,3}+h_{3,3}\right]$,

$x_{2,2}^{(2)}=p^{2}(1-p)\left[1-2 h_{1,2}+h_{2,2}\right]+p^{3}\left[1-2 h_{1,3}+h_{2,3}\right]$,

$x_{3,2}^{(2)}=x_{2,2}^{(2)}$,

$x_{1,1}^{(1)}=2 p^{2}(1-p)\left[1-2 h_{1,2}+h_{2,2}\right]$

$$
+p^{3}\left[1-h_{1,3}-h_{2,3}+h_{3,3}\right]
$$

$x_{2,1}^{(2)}=p^{2}(1-p)\left[h_{1,2}-h_{2,2}\right]+p^{2}(1-p)\left[1-2 h_{1,2}+h_{2,2}\right]$

$$
+p^{3}\left[h_{1,3}-h_{2,3}\right]
$$

$x_{2,1}^{(1)}=x_{11}^{(1)}$,

$x_{3,1}^{(2)}=x_{2,1}^{(2)}$,

$x_{3,1}^{(3)}=2 p^{2}(1-p)\left[h_{1,2}-h_{2,2}\right]+p^{3}\left[h_{2,3}-h_{3,3}\right]$,

$$
S=x_{1,1}^{(1)}
$$

where

$$
\begin{aligned}
& h_{1,3}=e^{-z_{1} x_{1,1}^{(1)}-z_{2}\left(2 x_{2,2}^{(2)}+2 x_{2,1}^{(2)}\right)-z_{3}\left(x_{3,3}^{(3)}+2 x_{3,2}^{(3)}+x_{3,1}^{(3)}\right)}, \\
& h_{2,3}=e^{-2 z_{1} x_{1,1}^{(1)}-z_{2}\left(3 x_{2,2}^{(2)}+4 x_{2,1}^{(2)}\right)-z_{3}\left(x_{3,3}^{(3)}+3 x_{3,2}^{(3)}+2 x_{3,1}^{(3)}\right)}, \\
& h_{3,3}=e^{-3 z_{1} x_{1,1}^{(1)}-z_{2}\left(3 x_{2,2}^{(2)}+6 x_{2,1}^{(2)}\right)-z_{3}\left(x_{3,3}^{(3)}+3 x_{3,2}^{(3)}+3 x_{3,1}^{(3)}\right)}, \\
& h_{1,2}=e^{-z_{1} x_{1,1}^{(1)}-z_{2}\left(x_{2,2}^{(2)}+x_{2,1}^{(2)}+x_{2,1}^{(1)}\right)-z_{3}\left(x_{3,2}^{(2)}+x_{3,1}^{(2)}\right)}, \\
& h_{2,2}=e^{-2 z_{1} x_{1,1}^{(1)}-z_{2}\left(x_{2,2}^{(2)}+2 x_{2,1}^{(2)}+2 x_{2,1}^{(1)}\right)-z_{3}\left(x_{3,2}^{(2)}+2 x_{3,1}^{(2)}\right)} .
\end{aligned}
$$

We note that, in the absence of overlap, i.e., for $z_{1}=z$, $z_{2}=0$ and $z_{3}=0$, Eqs. (33) reduce to Eqs. (17).

By defining a suitable order of the variables $x_{\mu, \nu}^{(\xi)}$, it is possible to introduce a vector $\mathbf{x}$ whose elements are the variables $x_{\mu, \nu}^{(\xi)}$, and to rewrite Eqs. (33) in a matrix form as

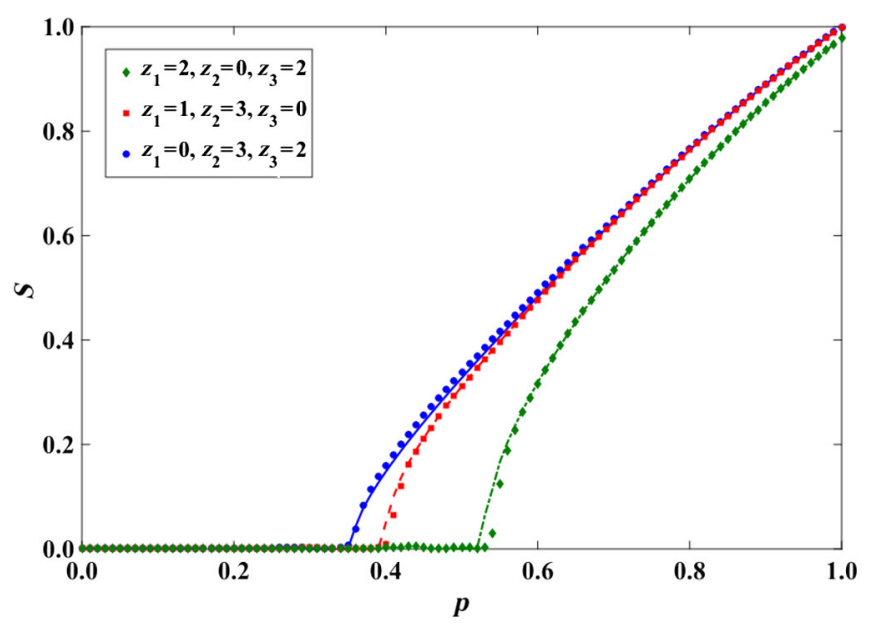

FIG. 9. Comparison between the simulation results of the RMCGC for a multiplex network with $M=3$ layers and Poisson multidegree distribution with $\left\langle k^{(1,1,1)}\right\rangle=c_{3},\left\langle k^{(1,1,0)}\right\rangle=$ $\left\langle k^{(1,0,1)}\right\rangle=\left\langle k^{(0,1,1)}\right\rangle=c_{2}$ and $\left\langle k^{(1,0,0)}\right\rangle=\left\langle k^{(0,1,0)}\right\rangle=\left\langle k^{(0,0,1)}\right\rangle=$ $c_{1}$ and the theoretic predictions over the same multilayer network ensemble. Data are shown for $c_{1}=0, c_{2}=3, c_{3}=2$ (blue circles), $c_{1}=1, c_{2}=3, c_{3}=0$ (red squares), and $c_{1}=2$, $c_{2}=0, c_{3}=2$ (green diamonds). The theoretical predictions are indicated with lines. The simulation results are performed on multilayer networks with $N=10^{4}$ nodes and are averaged over 20 multilayer network realizations. 


$$
\mathbf{G}(\mathbf{x})=\mathbf{0}
$$

The hybrid discontinuous phase transition (characterized by a square-root singularity) can be found by imposing that the system of Eqs. (35) is satisfied, together with the condition that the determinant of the Jacobian $\mathbf{J}$ of $\mathbf{G}(\mathbf{x})$ equals zero, that is,

$$
\mathbf{G}(\mathbf{x})=\mathbf{0} \quad \text { and } \quad \operatorname{det} \mathbf{J}=0 .
$$

Simulation results of the percolation process for multiplex networks in this ensemble are presented in Fig. 9, and they provide clear evidence of a perfect agreement with the theoretical prediction.

\section{CONCLUSIONS}

In this paper, we introduced and fully characterized an alternative percolation model for multiplex networks. The model serves to quantify the robustness of networks with redundant interdependencies. According to the model, interdependencies make a system more fragile than it would be by considering each layer independently. This fact is consistent with the original model used to study percolation in multiplex networks [4,21,23], and it is apparent from the fact that the transition is abrupt for any number of network layers considered in the interdependent model. On the other hand, redundancy of interdependencies across multiple layers favors system robustness, as the height of the discontinuous jump and the location of the transition point decrease as the number of layers increases. This is a fundamental difference with respect to the model currently adopted to study the robustness of multiplex networks and multilayer networks, in general, where instead increasing the number of layers generates more and more fragile networks [23,26-28].

To characterize the model, we deployed a comprehensive theoretical approach based on message passing. Our theory is valid for arbitrary multiplex network topologies as long as they are locally treelike. The theory is further developed in the context of ensembles of synthetic network models to analyze properties of the percolation transition emerging in the new model and to perform systematic comparisons, and emphasize fundamental differences, with the percolation model introduced in Ref. [4].

We remark that ours is not a definitive model, but it represents only a good starting point towards a more realistic description of real multiplex systems. For instance, we do not expect to observe a qualitative change of the results if the redundancy is weaker, and let us say a node is damaged only if less than two interdependent nodes are functioning. However, our approach is purely structural, and in many situations, forgetting about intrinsic dynamical features may not be appropriate. The primary role of our model is to emphasize the importance of redundancy or complementarity in multilayer networks, an obvious, yet neglected feature of many real systems. In several realistic settings, in fact, system robustness is augmented by the addition of new layers of interactions, as these layers are indeed created to provide backup options. For example, adding a new mode of transportation in a preexisting multimodal transportation system should make the system more reliable against eventual failures. Similarly, in a living organism, the development of new types of interactions among constituents should increase the stability of the same organism against possible mutations. In the current setting, the model assumes that the functioning of individual nodes requires that nodes are correctly operating on at least two interdependent layers. However, the model can be generalized to deal with a variable number of minimal functioning layers to describe more realistic scenarios in specific situations of interest.

\section{ACKNOWLEDGMENTS}

F. R. acknowledges support from the National Science Foundation (Grant No. CMMI-1552487) and the U.S. Army Research Office (Grant No. W911NF-16-1-0104).

[1] S. Boccaletti, G. Bianconi, R. Criado, C. I. del Genio, J. Gómez-Gardeñes, M. Romance, I. Sendiña-Nadal, Z. Wang, and M. Zanin, The Structure and Dynamics of Multilayer Networks, Phys. Rep. 544, 1 (2014).

[2] M. Kivelä, A. Arenas, M. Barthélemy, J. P. Gleeson, Y. Moreno, and M. A. Porter, Multilayer Networks, J. Complex Netw. 2, 203 (2014).

[3] K.-M. Lee, B. Min, and K.-I. Goh, Towards Real-World Complexity: An Introduction to Multiplex Networks, Eur. Phys. J. B 88, 48 (2015).

[4] S. V. Buldyrev, R. Parshani, G. Paul, H. E. Stanley, and S. Havlin, Catastrophic Cascade of Failures in Interdependent Networks, Nature (London) 464, 1025 (2010).

[5] J. Gao, S. V. Buldyrev, H. E. Stanley, and S. Havlin, Networks Formed from Interdependent Networks, Nat. Phys. 8, 40 (2012).

[6] M. Szell, R. Lambiotte, and S. Thurner, Multirelational Organization of Large-Scale Social Networks in an Online World, Proc. Natl. Acad. Sci. U.S.A. 107, 13636 (2010).

[7] G. Menichetti, D. Remondini, P. Panzarasa, R. J. Mondragón, and G. Bianconi, Weighted Multiplex Networks, PLoS One 9 , e97857 (2014).

[8] A. Cardillo, J. Gómez-Gardeñes, M. Zanin, M. Romance, D. Papo, F. del Pozo, and S. Boccaletti, Emergence of Network Features from Multiplexity, Sci. Rep. 3, 1344 (2013).

[9] M. De Domenico, A. Solé-Ribalta, S. Gómez, and A. Arenas, Navigability of Interconnected Networks under Random Failures, Proc. Natl. Acad. Sci. U.S.A. 111, 8351 (2014).

[10] V. Nicosia and V. Latora, Measuring and Modeling Correlations in Multiplex Networks, Phys. Rev. E 92, 032805 (2015). 
[11] J. Menche, A. Sharma, M. Kitsak, S. D. Ghiassian, M. Vidal, J. Loscalzo, and A.-L. Barabási, Uncovering Disease-Disease Relationships through the Incomplete Interactome, Science 347, 1257601 (2015).

[12] E. Bullmore and O. Sporns, Complex Brain Networks: Graph Theoretical Analysis of Structural and Functional Systems, Nat. Rev. Neurosci. 10, 186 (2009).

[13] S. D. S. Reis, Y. Hu, A. Babino, J. S. Andrade Jr., S. Canals, M. Sigman, and H. A. Makse, Avoiding Catastrophic Failure in Correlated Networks of Networks, Nat. Phys. 10, 762 (2014).

[14] M. De Domenico, A. Solé-Ribalta, E. Cozzo, M. Kivelä, Y. Moreno, M. A. Porter, S. Gómez, and A. Arenas, Mathematical Formulation of Multilayer Networks, Phys. Rev. X 3, 041022 (2013).

[15] P. J. Mucha, T. Richardson, K. Macon, M. A. Porter, and J. P. Onnela, Community Structure in Time-Dependent, Multiscale, and Multiplex Networks, Science 328, 876 (2010).

[16] G. Bianconi, Statistical Mechanics of Multiplex Networks: Entropy and Overlap, Phys. Rev. E 87, 062806 (2013).

[17] N. Azimi-Tafreshi, J. Gómez-Gardeñes, and S. N. Dorogovtsev, k-Core Percolation on Multiplex Networks, Phys. Rev. E 90, 032816 (2014).

[18] G. J. Baxter, S. N. Dorogovtsev, J. F. F. Mendes, and D. Cellai, Weak Percolation on Multiplex Networks, Phys. Rev. E 89, 042801 (2014).

[19] A. Hackett, D. Cellai, S. Gómez, A. Arenas, and J. P. Gleeson, Bond Percolation on Multiplex Networks, Phys. Rev. X 6, 021002 (2016).

[20] S. Watanabe and Y. Kabashima, Cavity-Based Robustness Analysis of Interdependent Networks: Influences of Intranetwork and Internetwork Degree-Degree Correlations, Phys. Rev. E 89, 012808 (2014).

[21] G. J. Baxter, S. N. Dorogovtsev, A. V. Goltsev, and J. F. F. Mendes, Avalanche Collapse of Interdependent Networks, Phys. Rev. Lett. 109, 248701 (2012).

[22] B. Min, S. D. Yi, K.-M. Lee, and K.-I. Goh, Network Robustness of Multiplex Networks with Interlayer Degree Correlations, Phys. Rev. E 89, 042811 (2014).

[23] S.-W. Son, G. Bizhani, C. Christensen, P. Grassberger, and M. Paczuski, Percolation Theory on Interdependent Networks Based on Epidemic Spreading, Europhys. Lett. 97, 16006 (2012).

[24] R. Parshani, C. Rozenblat, D. Ietri, C. Ducruet, and S. Havlin, Inter-Similarity between Coupled Networks, Europhys. Lett. 92, 68002 (2010).

[25] R. Parshani, S. V. Buldyrev, and S. Havlin, Interdependent Networks: Reducing the Coupling Strength Leads to a Change from a First to Second Order Percolation Transition, Phys. Rev. Lett. 105, 048701 (2010).

[26] D. Cellai, S. N. Dorogovtsev, and G.Bianconi, Message Passing Theory for Percolation Models on Multiplex Networks with Link Overlap, Phys. Rev. E 94, 032301 (2016).

[27] G. Bianconi, S. N. Dorogovtsev, and J. F. F. Mendes, Mutually Connected Component of Networks of Networks with Replica Nodes, Phys. Rev. E 91, 012804 (2015).
[28] G. Bianconi and S. N. Dorogovtsev, Multiple Percolation Transitions in a Configuration Model of a Network of Networks, Phys. Rev. E 89, 062814 (2014).

[29] F. Radicchi and A. Arenas, Abrupt Transition in the Structural Formation of Interconnected Networks, Nat. Phys. 9, 717 (2013).

[30] E. Leicht and R. D'Souza, Percolation on Interacting Networks, arXiv:0907.0894.

[31] K.-M. Lee, J. Y. Kim, W. Cho, K.-I. Goh, and I.-M. Kim, Correlated Multiplexity and Connectivity of Multiplex Random Networks, New J. Phys. 14, 033027 (2012).

[32] J. Shao, S. V. Buldyrev, S. Havlin, and H. E. Stanley, Cascade of Failures in Coupled Network Systems with Multiple Support-Dependence Relations, Phys. Rev. E 83, 036116 (2011).

[33] S. N. Dorogovtsev, A. Goltsev, and J. F. F. Mendes, Critical Phenomena in Complex Networks, Rev. Mod. Phys. 80, 1275 (2008).

[34] B. Karrer, M. E. J. Newman, and L. Zdeborová, Percolation on Sparse Networks, Phys. Rev. Lett. 113, 208702 (2014).

[35] M. Mezard and A. Montanari, Information, Physics and Computation (Oxford University Press, Oxford, 2009).

[36] A. K. Hartmann and M. Weigt, Phase Transitions in Combinatorial Optimization Problems (Wiley-VCH, Weinheim, 2005).

[37] G. J. Baxter, G. Bianconi, R. A. da Costa, S. N. Dorogovtsev, and J. F. F. Mendes, Correlated Link Overlaps in Multiplex Networks, Phys. Rev. E 94, 012303 (2016).

[38] D. Cellai, E. López, J. Zhou, J. P. Gleeson, and G. Bianconi, Percolation in Multiplex Networks with Overlap, Phys. Rev. E 88, 052811 (2013).

[39] F. Radicchi, Percolation in Real Interdependent Networks, Nat. Phys. 11, 597 (2015).

[40] B. Min, S. Lee, K.-M. Lee, and K.-I. Goh, Link Overlap, Viability, and Mutual Percolation in Multiplex Networks, Chaos Solitons Fractals 72, 49 (2015).

[41] F. Radicchi and C. Castellano, Beyond the Locally Treelike Approximation for Percolation on Real Networks, Phys. Rev. E 93, 030302(R) (2016).

[42] S. Gómez, A. Díaz-Guilera, J. Gómez-Gardeñes, C. J. Perez-Vicente, Y. Moreno, and A. Arenas, Diffusion Dynamics on Multiplex Networks, Phys. Rev. Lett. 110, 028701 (2013).

[43] M. De Domenico, C. Granell, M. A. Porter, and A. Arenas, The Physics of Spreading Processes in Multilayer Networks, Nat. Phys. 12, 901 (2016).

[44] J. Gao, S. V. Buldyrev, S. Havlin, and H. E. Stanley, Robustness of a Network Formed by $n$ Interdependent Networks with a One-to-One Correspondence of Dependent Nodes, Phys. Rev. E 85, 066134 (2012).

[45] J. Gao, S. V. Buldyrev, S. Havlin, and H. E. Stanley, Robustness of a Network of Networks, Phys. Rev. Lett. 107, 195701 (2011).

[46] See Supplemental Material at http://link.aps.org/ supplemental/10.1103/PhysRevX.7.011013 for the complete framework that describes the redundant percolation model on multiplex networks with edge overlap. 